\title{
Article
}

\section{Common Independence in Graphs}

\author{
Magda Dettlaff ${ }^{1}$, Magdalena Lemańska ${ }^{1, *}$ and Jerzy Topp ${ }^{2}$ \\ 1 Technical Physics and Applied Mathematics Department, Gdańsk University of Technology, \\ 80-803 Gdańsk, Poland; magda.dettlaff@pg.edu.pl \\ 2 Institute of Applied Informatics, The State University of Applied Sciences in Elbląg, 82-300 Elbląg, Poland; \\ j.topp@pwsz.elblag.pl \\ * Correspondence: magdalena.lemanska@pg.edu.pl
}

\section{check for} updates

Citation: Dettlaff, M.; Lemańska, M.; Topp, J. Common Independence in Graphs. Symmetry 2021, 13, 1411. https://doi.org/10.3390/sym13081411

Academic Editor: Egon Schulte

Received: 7 July 2021

Accepted: 27 July 2021

Published: 2 August 2021

Publisher's Note: MDPI stays neutral with regard to jurisdictional claims in published maps and institutional affiliations.

Copyright: (c) 2021 by the authors. Licensee MDPI, Basel, Switzerland. This article is an open access article distributed under the terms and conditions of the Creative Commons Attribution (CC BY) license (https:// creativecommons.org/licenses/by/ $4.0 /)$.

\begin{abstract}
The cardinality of a largest independent set of $G$, denoted by $\alpha(G)$, is called the independence number of $G$. The independent domination number $i(G)$ of a graph $G$ is the cardinality of a smallest independent dominating set of $G$. We introduce the concept of the common independence number of a graph $G$, denoted by $\alpha_{c}(G)$, as the greatest integer $r$ such that every vertex of $G$ belongs to some independent subset $X$ of $V_{G}$ with $|X| \geq r$. The common independence number $\alpha_{c}(G)$ of $G$ is the limit of symmetry in $G$ with respect to the fact that each vertex of $G$ belongs to an independent set of cardinality $\alpha_{\mathcal{c}}(G)$ in $G$, and there are vertices in $G$ that do not belong to any larger independent set in $G$. For any graph $G$, the relations between above parameters are given by the chain of inequalities $i(G) \leq \alpha_{\mathcal{c}}(G) \leq \alpha(G)$. In this paper, we characterize the trees $T$ for which $i(T)=\alpha_{\mathcal{c}}(T)$, and the block graphs $G$ for which $\alpha_{c}(G)=\alpha(G)$.
\end{abstract}

Keywords: independence number; domination number; independence domination number; common independence number

MSC: [2010] 05C69

\section{Introduction}

For notation and graph theory terminology we, in general, follow [1]. Specifically, let $G=\left(V_{G}, E_{G}\right)$ be a graph with vertex set $V_{G}$ and edge set $E_{G}$. If $A$ and $B$ are disjoint sets of vertices of $G$, then we denote by $E_{G}(A, B)$ the set of edges in $G$ joining a vertex in $A$ with a vertex in $B$. For a vertex $v$ of $G$, its neighborhood, denoted by $N_{G}(v)$, is the set of all vertices adjacent to $v$, and the cardinality of $N_{G}(v)$, denoted by $d_{G}(v)$, is called the degree of $v$. The closed neighborhood of $v$, denoted by $N_{G}[v]$, is the set $N_{G}(v) \cup\{v\}$. In general, for a subset $X \subseteq V_{G}$ of vertices, the neighborhood of $X$, denoted by $N_{G}(X)$, is defined to be $\cup_{v \in X} N_{G}(v)$, and the closed neighborhood of $X$, denoted by $N_{G}[X]$, is the set $N_{G}(X) \cup X$. A vertex of degree 0 is said to be isolated in $G$, while a vertex of degree one in $G$ is called a leaf of $G$. The set of all leaves of $G$ is denoted by $L_{G}$. We define a pendant edge of a graph to be an edge incident with a leaf. The corona of graphs $H$ and $F$ is a graph $H \circ F$ resulting from the disjoint union of $H$ and $\left|V_{H}\right|$ copies of $F$ in which each vertex $v$ of $H$ is adjacent to all vertices of the copy of $F$ corresponding to $v$. The corona $H \circ K_{1}$, in particular, is the graph obtained from $H$ by adding exactly one pendant edge to each vertex of $H$. A graph $G$ is said to be a corona if $G$ is the corona $H \circ K_{1}$ of some graph $H$. It is obvious that a corona is a graph in which each vertex is a leaf or it is adjacent to exactly one leaf.

We denote the path, cycle, and complete graph on $n$ vertices by $P_{n}, C_{n}$, and $K_{n}$, respectively. The complete bipartite graph with one partite set of size $n$ and the other of size $m$ is denoted by $K_{n, m}$. A star is the tree $K_{1, k}$ for some $k \geq 1$. For $k, l \geq 1$, a double star $S(k, l)$ is the tree with exactly two vertices that are not leaves, one of which has $k$ leaf neighbors and the other $l$ leaf neighbors.

A vertex $v$ is called a simplicial vertex of $G$ if $N_{G}[v]$ is a complete graph, while it is a cut vertex if $G-v$ is disconnected. A block of a graph $G$ is a maximal connected subgraph of $G$ 
without its own cut vertices. We say that $G$ is a block graph if every block of $G$ is a complete graph (equivalently, every vertex of $G$ is a simplicial or a cut vertex). A block of a block graph $G$ is called a simplex if it contains at least one simplicial vertex of $G$, while it is an end block if it contains at most one cut vertex of $G$.

A subset $D$ of $V_{G}$ is called a dominating set of $G$ if every vertex belonging to $V_{G}-D$ is adjacent to at least one vertex in $D$. A subset $I$ of $V_{G}$ is said to be independent if no two vertices belonging to $I$ are adjacent in $G$. The cardinality of a largest (i.e., maximum) independent set of $G$, denoted by $\alpha(G)$, is called the independence number of $G$. Every largest independent set of a graph is called an $\alpha$-set of the graph. The independent domination number of $G$, denoted by $i(G)$, is the cardinality of a smallest independent dominating set of $G$ (or equivalently, the cardinality of a minimum maximal independent set of vertices in $G$ ). The study of independent sets in graphs was begun by Berge [2,3] and Ore [4]. In 2013 Goddard and Henning published an article [5] that summarized results on independence domination in graphs. It is obvious that $i(G) \leq \alpha(G)$ for any graph $G$. A graph $G$ is a well-covered graph if $i(G)=\alpha(G)$. Equivalently, $G$ is well-covered if every maximal independent set of $G$ is a maximum independent set of $G$. The concept of well-covered graphs was introduced by Plummer [6] and extensively studied in many papers. We refer the reader to the excellent (but already old) survey on well-covered graphs by Plummer [7].

Now, between the integers $i(G)$ and $\alpha(G)$, we insert another integer concerning the existence and cardinality of independent sets in $G$. Formally, we introduce the concept of the common independence number of a graph $G$, denoted by $\alpha_{c}(G)$, as the greatest integer $r$ such that every vertex of $G$ belongs to some independent subset $X$ of $V_{G}$ with $|X| \geq r$. Thus, the common independence number of a graph $G$ refers to numbers of mutually independent vertices of $G$, and it emphasizes the notion of the individual independence of a vertex of $G$ from other vertices of $G$. The common independence number $\alpha_{c}(G)$ of $G$ is the limit of symmetry in $G$ with respect to the fact that each vertex of $G$ belongs to an independent set of cardinality $\alpha_{c}(G)$ in $G$, and there are vertices in $G$ that do not belong to any larger independent set in G. For possible applications of the three parameters $i(G), \alpha(G)$, and $\alpha_{c}(G)$ we refer the reader to the newest survey by Majeed and Rauf [8] on different applications of graph theory in computer science and social networks. It follows immediately from the above definitions (see also Proposition 1) that $i(G) \leq \alpha_{\mathcal{c}}(G) \leq \alpha(G)$ for any graph $G$. In the following section, we present the first properties of the common independence number. Then, in the next two sections, we characterize the family of trees $T$ for which $i(T)=\alpha_{c}(T)$, and the family of block graphs $G$ such that $\alpha_{\mathcal{c}}(G)=\alpha(G)$, respectively. (We remark that the family of well-covered graphs is a proper subfamily of each of the previously mentioned families.)

\section{Preliminaries}

For our studies of the common independence number of a graph, we begin from straightforward propositions and simple examples.

Proposition 1. For every graph $G$ we have $i(G) \leq \alpha_{c}(G) \leq \alpha(G)$.

Proof. Certainly, we have $\alpha_{c}(G) \leq \alpha(G)$, since, as it follows from the definition of $\alpha(G)$, every independent set of vertices of $G$ has at most $\alpha(G)$ vertices. On the other hand, for every $v \in V_{G}$, let $I_{v}$ be any maximal independent subset of $V_{G}$ that contains $v$. The maximality of $I_{v}$ implies that $\left|I_{v}\right| \geq i(G)$. Consequently, $\alpha_{c}(G) \geq i(G)$.

Proposition 2. If $G$ is a graph, then $\alpha_{c}(G)=1$ if and only if $i(G)=1$.

Proof. If $i(G)=1$, then $G$ has a vertex $v$ that is adjacent to every other vertex of $G$. This implies that $\{v\}$ is the only independent set in $G$ that contains $v$. Hence $\alpha_{c}(G)=1$. On the other hand, if $\alpha_{\mathcal{c}}(G)=1$, then $i(G)=1$ by Proposition 1 . 
Proposition 3. If $G$ is a non-empty graph, then $\alpha_{c}(G)=\min \left\{\alpha\left(G-N_{G}[v]\right): v \in V_{G}\right\}+1$ and $i(G)=\min \left\{i\left(G-N_{G}[v]\right): v \in V_{G}\right\}+1$, assuming that $i(H)=\alpha(H)=0$ if $H$ is an empty graph, that is, a graph without vertices.

Proof. Both equalities are obvious if $G$ has a vertex $v$ such that $N_{G}[v]=V_{G}$ (in this case $G-N_{G}[v]$ is an empty graph). Assume now $G-N_{G}[v]$ is non-empty for every $v \in V_{G}$. Let $I_{v}$ be a largest independent set in $G-N_{G}[v]$. Then $I_{v} \cup\{v\}$ is a largest independent set in $G$ that contains $v$. From this and from the definition of the common independence number it follows that $\alpha_{c}(G)=\min \left\{\alpha\left(G-N_{G}[v]\right): v \in V_{G}\right\}+1$. Now let $J_{v}$ be a minimum independent dominating set in $G-N_{G}[v]$. Then $J_{v} \cup\{v\}$ is a maximal independent set in $G$, which implies $i(G) \leq i\left(G-N_{G}[v]\right)+1$. Assume now $D$ is a minimum independent dominating set in $G$ and let $v^{\prime} \in D$. Then $D-\left\{v^{\prime}\right\}$ is a minimum independent dominating set in $G-N_{G}[v]$ and $i\left(G-N_{G}\left[v^{\prime}\right]\right)=i(G)-1$. Hence, $i(G)=\min \left\{i\left(G-N_{G}[v]\right): v \in\right.$ $\left.V_{G}\right\}+1$.

To better recognize connections between the independent domination number $i(G)$, the common independence number $\alpha_{c}(G)$, and the independence number $\alpha(G)$, we begin with simple examples. It is obvious that $i\left(K_{n}\right)=\alpha_{c}\left(K_{n}\right)=\alpha\left(K_{n}\right)=1$ for every positive integer $n$. Similarly, if $m$ and $n$ are positive integers and $m \leq n$, then $i\left(K_{m, n}\right)=\alpha_{c}\left(K_{m, n}\right)=$ $m \leq \alpha\left(K_{m, n}\right)=n$. It is no problem to observe that $i\left(P_{n}\right)=\lceil n / 3\rceil, \alpha_{c}\left(P_{n}\right)=\lfloor n / 2\rfloor$, and $\alpha\left(P_{n}\right)=\lceil n / 2\rceil$. Consequently, $i\left(P_{n}\right)<\alpha_{c}\left(P_{n}\right)<\alpha\left(P_{n}\right)$ if $n=2 k+1$ and $k \geq 4$. On the other hand we have $i\left(C_{n}\right)=\lfloor n / 3\rfloor \leq \alpha_{c}\left(C_{n}\right)=\lceil(n-1) / 2\rceil=\lfloor n / 2\rfloor=\alpha\left(C_{n}\right)$ if $n \geq 3$. Let $G_{k}$ be a graph obtained from the corona $K_{1, k} \circ \bar{K}_{2}$ by inserting a new vertex into each non-pendant edge of $K_{1, k} \circ \bar{K}_{2}$, see Figure 1 . Now it is easy to see that $i\left(G_{k}\right)=k+1$, $\alpha_{c}\left(G_{k}\right)=2 k+1, \alpha\left(G_{k}\right)=3 k+2$, and $i\left(G_{k}\right)<\alpha_{c}\left(G_{k}\right)<\alpha\left(G_{k}\right)$ if $k \geq 1$. Similarly, if $S^{*}(k, l)$ denotes a graph obtained from the double star $S(k, l)$ by inserting a new vertex into its only non-pendant edge, then it is obvious that $i\left(S^{*}(k, l)\right)=2, \alpha_{c}\left(S^{*}(k, l)\right)=\min \{k, l\}+1$, and $\alpha\left(S^{*}(k, l)\right)=k+l+1$. From these examples it follows that the differences between numbers $i(G), \alpha_{c}(G)$, and $\alpha(G)$ can be arbitrarily large.

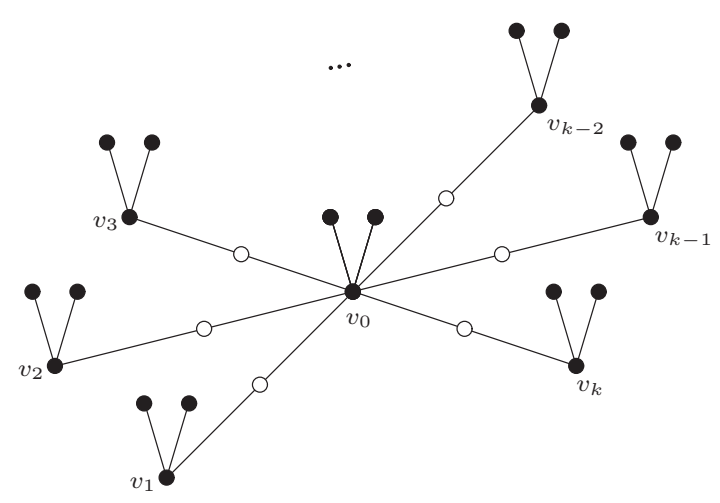

Figure 1. Graph $G_{k}$.

The aim of the next theorem is to show for which positive integers $m, n$, and $p$ satisfying the inequalities $m \leq n \leq p$ there exists a graph $G$ such that $i(G)=m, \alpha_{c}(G)=n$, and $\alpha(G)=p$. This theorem again shows that the difference between the independence number and the common independence number as well as the difference between the common independence number and the independent domination number of a graph can be arbitrarily large.

Theorem 1. For integers $m, n$, and $p$ there exists a graph $G$ with $i(G)=m, \alpha_{c}(G)=n$, and $\alpha(G)=p$ if and only if $m=n=1 \leq p$ or $2 \leq m \leq n \leq p$.

Proof. Since the necessity is obvious from Propositions 1 and 2, we need only provide constructions to establish sufficiency. 
If $m=n=1 \leq p$, then for $K_{1, p}$ we have $i\left(K_{1, p}\right)=\alpha_{c}\left(K_{1, p}\right)=1$ and $\alpha\left(K_{1, p}\right)=p$. Thus assume that $2 \leq m \leq n \leq p$, and let $s=\lfloor m / 2\rfloor, t=m-s(=\lceil m / 2\rceil)$. Let $H_{1}, H_{2}, H_{3}$, and $H_{4}$ be disjoint totally disconnected graphs of order $s, p-t, n-s$, and $t$, respectively. Now, let $G$ be a new graph constructed from the union $H_{1} \cup H_{2} \cup H_{3} \cup H_{4}$ by adding all possible edges between the vertices of $H_{i}$ and $H_{i+1}, i=1,2,3$. It is easy to observe that the sets $V_{H_{1}} \cup V_{H_{4}}, V_{H_{1}} \cup V_{H_{3}}$, and $V_{H_{2}} \cup V_{H_{4}}$ of cardinality $m, n$, and $p$, respectively, are the only maximal independent sets of $G$. Consequently, $i(G)=m$ and $\alpha(G)=p$. In addition, because every vertex of $G$ belongs to an independent set of cardinality at least $n$ and no vertex in $V_{H_{1}} \cup V_{H_{3}}$ belongs to an independent set of cardinality greater than $n$, we have $\alpha_{c}(G)=n$. This completes the proof.

\section{Graphs $G$ with $\alpha(G)=\alpha_{c}(G)$}

In this section we consider graphs $G$ for which $\alpha(G)=\alpha_{c}(G)$; in particular we provide a constructive characterization of block graphs $G$ for which $\alpha(G)=\alpha_{\mathcal{c}}(G)$. It follows from the next proposition that such graphs form the class of the $\alpha$-excellent graphs, that is, the class of graphs $G$ in which every vertex belongs to some largest independent set of $G$. Thus, we are interested in characterizations of the $\alpha$-excellent graphs. The $\alpha$-excellent trees were already studied in $[9,10]$. We extend their characterizations to the $\alpha$-excellent block graphs, and we present some additional properties of the $\alpha$-excellent trees.

Proposition 4. For a graph $G$ is $\alpha_{\mathcal{c}}(G)=\alpha(G)$ if and only if $G$ is an $\alpha$-excellent graph.

Proof. Assume that $G$ is an $\alpha$-excellent graph. Then, by definition, every vertex of $G$ belongs to an independent set of cardinality $\alpha(G)$ in $G$, and, therefore, $\alpha_{c}(G) \geq \alpha(G)$. From this and from Proposition 1 it follows that $\alpha_{c}(G)=\alpha(G)$.

On the other hand, by definition, every vertex of $G$ belongs to an independent set of cardinality (at least) $\alpha_{c}(G)$. Thus, if $\alpha_{c}(G)=\alpha(G)$, then every vertex of $G$ belongs to an independent set of cardinality $\alpha(G)$, and $G$ is $\alpha$-excellent.

In order to state and prove our characterization of the $\alpha$-excellent block graphs, we present additional definitions and some preliminary results that we will need while proving the next two theorems. Let $K_{n}$ be a complete graph of order $n \geq 1$ with vertex set $\left\{v_{1}, \ldots, v_{n}\right\}$. If $k_{1}, \ldots, k_{n}$ are positive integers, then by $K_{n}^{k_{1}, \ldots, k_{n}}$ we denote a graph obtained from the disjoint union of the complete graphs $K_{n}, K_{k_{1}}, \ldots, K_{k_{n}}$ by joining each vertex $v_{i}$ of $K_{n}$ with each vertex of $K_{k_{i}}$ for $i=1, \ldots, n$. The graph $K_{n}^{k_{1}, \ldots, k_{n}}$ is said to be a general corona of $K_{n}$ and the subgraph of $K_{n}^{k_{1}, \ldots, k_{n}}$ induced by the vertices $v_{1}, \ldots, v_{n}$ is called the body of $K_{n}^{k_{1}, \ldots, k_{n}}$. It is obvious that $K_{n}^{k_{1}, \ldots, k_{n}}$ has the property stated in the next observation.

Observation 1. A general corona $K_{n}^{k_{1}, \ldots, k_{n}}$ is a well-covered graph, and $\alpha\left(K_{n}^{k_{1}, \ldots, k_{n}}\right)=n$. In addition, $K_{n}^{k_{1}, \ldots, k_{n}}$ is a tree if and only if either $n=k_{1}=1$ or $n=2$ and $k_{1}=k_{2}=1$.

Let $\mathcal{F}$ be the family of graphs that: (1) contains every complete graph of order at least 2; and (2) is closed under attaching general coronas, that is, if a graph $G^{\prime}$ belongs to $\mathcal{F}$ and $H=K_{n}^{k_{1}, \ldots, k_{n}}$ is a general corona, then to $\mathcal{F}$ belongs every graph obtained from the disjoint union $G^{\prime} \cup H$ by adding $n$ edges that join one vertex of $G^{\prime}$ with the vertices forming the body of $H$. By $\mathcal{E}$ we denote the family (defined in [9]) of all trees belonging to $\mathcal{F}$. Thus, $K_{2}$ belongs to $\mathcal{E}$, and, if a graph $G^{\prime}$ belongs to $\mathcal{E}$ and $K$ is a complete graph of order 2 , then to $\mathcal{E}$ belongs every graph obtained from the disjoint union $G^{\prime} \cup K$ by adding exactly one edge that joins a vertex of $G^{\prime}$ with a vertex of $K$.

It is clear from the above definition that every graph belonging to the family $\mathcal{F}$ is a block graph. Figure 2 shows a block graph $G$ belonging to $\mathcal{F}$ and a tree $T$ that belongs to the subfamily $\mathcal{E}$ of $\mathcal{F}$. 

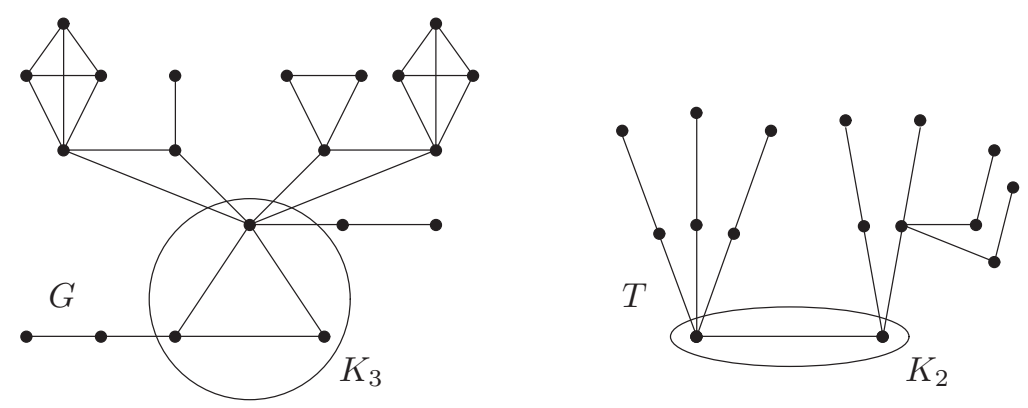

Figure 2. Graphs $G \in \mathcal{F}$ and $T \in \mathcal{E}$.

Proposition 5. Let $G^{\prime}$ be a connected graph of order at least 2. If $G$ is a graph obtained from $G^{\prime}$ by attaching a general corona $K_{n}^{k_{1}, \ldots, k_{n}}$ to a single vertex of $G^{\prime}$, then $\alpha(G)=\alpha\left(G^{\prime}\right)+n$. In addition, $G$ is an $\alpha$-excellent graph if and only if $G^{\prime}$ is an $\alpha$-excellent graph.

Proof. Assume that $\left\{v_{1}, \ldots, v_{n}\right\}$ is the vertex set of the body of $H=K_{n}^{k_{1}, \ldots, k_{n}}$, and assume that $H$ is attached to a vertex $v$ of $G^{\prime}$. Let $S=\left\{v_{1}^{\prime}, \ldots, v_{n}^{\prime}\right\}$ be a set of simplicial vertices of $H$, where each $v_{i}^{\prime}$ is adjacent to $v_{i}(i=1, \ldots, n)$. It is apparent that if $I$ is an $\alpha$-set of $G^{\prime}$, then $I \cup S$ is an independent set of $G$, and therefore $\alpha(G) \geq|I \cup S|=\alpha\left(G^{\prime}\right)+n$. Thus assume that $J$ is an $\alpha$-set of $G$. Then $J \cap V_{G^{\prime}}$ and $J \cap V_{H}$ are independent sets of $G^{\prime}$ and $H$, respectively. In addition it follows from Observation 1 that $J \cap V_{H}$ is an $\alpha$-set of $H$. Hence $\alpha(G)=|J|=\left|J \cap V_{G^{\prime}}\right|+\left|J \cap V_{H}\right|=\left|J \cap V_{G^{\prime}}\right|+n \leq \alpha\left(G^{\prime}\right)+n$, and so $\alpha(G)=\alpha\left(G^{\prime}\right)+n$.

It remains to prove that $G$ is $\alpha$-excellent if and only if $G^{\prime}$ is $\alpha$-excellent. Assume first that $G$ is $\alpha$-excellent. Let $x$ be a vertex of $G^{\prime}$, and let $I_{x}$ be an $\alpha$-set of $G$ that contains $x$. Since $I_{x}$ has at most $n$ vertices in $H$ (by Observation 1), $I_{x}$ and has at least $\alpha(G)-n=\alpha\left(G^{\prime}\right)$ vertices in $G^{\prime}$ and $x \in I_{x}$. This implies that $G^{\prime}$ is an $\alpha$-excellent graph.

Now assume that $G^{\prime}$ is $\alpha$-excellent. Let $y$ be a vertex of $G$. If $y$ belongs to $V_{G^{\prime}}$, and if $I_{y}$ is an $\alpha$-set of $G^{\prime}$ containing $y$, then $I_{y} \cup S$ is an $\alpha$-set of $G$ containing $y$. Thus assume that $y \in V_{H}$. If $y$ is a simplicial vertex of $G$ belonging to $H$, then without loss of generality we may assume that $y \in S$. Then $I \cup S$ is an $\alpha$-set of $G$ containing $y$, where $I$ is any $\alpha$-set of $G^{\prime}$. If $y$ is in a body of $H$, say $y=v_{i}$ (for some $i \in\{1, \ldots, n\}$ ), then $I_{u} \cup\left(S-\left\{v_{i}^{\prime}\right\}\right) \cup\left\{v_{i}\right\}$ is an $\alpha$-set of $G$ containing $y$, where $u$ is any neighbor of $v$ in $G^{\prime}$ and $I_{u}$ an $\alpha$-set of $G^{\prime}$ containing $u$.

We are now in position to prove the main result of this section, a constructive characterization of the $\alpha$-excellent block graphs.

Theorem 2. Let $G$ be a block graph of order $n \geq 2$. Then the following statements are equivalent:

(a) $G \in \mathcal{F}$.

(b) $G$ is $\alpha$-excellent graph.

(c) $\alpha_{c}(G)=\alpha(G)$.

Proof. The implication $(\mathrm{a}) \Rightarrow(\mathrm{b})$ is obvious from Proposition 5. The statements (b) and (c) are equivalent by Proposition 4 . Thus it suffices to prove the implication $(\mathrm{c}) \Rightarrow(\mathrm{a})$.

Assume that $G$ is a block graph of order at least 2 with $n \geq 1$ blocks and $\alpha(G)=\alpha_{c}(G)$. We use induction on $n$ to show that $G \in \mathcal{F}$. If $n=1$, then $G$ is a complete graph, say $G=K_{m}(m \geq 2)$, and certainly $G \in \mathcal{F}$. Let $G$ be a block graph with $n \geq 2$ blocks and assume that every block graph $G^{\prime}$ belongs to $\mathcal{F}$ if $\alpha\left(G^{\prime}\right)=\alpha_{c}\left(G^{\prime}\right)$ and $G^{\prime}$ has $n^{\prime}$ blocks, where $1 \leq n^{\prime}<n$. We first establish the following claim.

Claim 1. All simplices of $G$ are pairwise vertex-disjoint.

Proof. Suppose that $S_{1}$ and $S_{2}$ are two distinct simplices of $G$ containing a common vertex $v$. Let $I_{v}$ be a largest independent set of $G$ that contains $v$. Then $\alpha(G) \geq\left|I_{v}\right| \geq \alpha_{c}(G)=\alpha(G)$. 
On the other hand, let $x$ and $y$ be two simplicial vertices belonging to $S_{1}$ and $S_{2}$, respectively. Then, since $x$ is not adjacent to $y$ and neither $x$ nor $y$ is adjacent to any vertex in $I_{v}-\{v\}$, the set $\left(I_{v}-\{v\}\right) \cup\{x, y\}$ is an independent set of $G$ and $\left|\left(I_{v}-\{v\}\right) \cup\{x, y\}\right|>\left|I_{v}\right|=\alpha(G)$, a contradiction which completes the proof of our claim.

Claim 1 implies that the diameter $d$ of $G$ is greater than 2 . Let $P=\left(u_{0}, u_{1}, \ldots, u_{d}\right)$ be a longest path without chords in $G$. Let $B_{i}$ be that block of $G$ which contains $u_{i}$ and $u_{i+1}, i=0, \ldots, d-1$. The choice of $P$ and Claim 1 imply that the blocks $B_{0}, \ldots, B_{d-1}$ are distinct, $u_{1}, \ldots, u_{d-1}$ are cut vertices, while $u_{0}$ and $u_{d}$ are simplicial vertices belonging to the end blocks $B_{0}$ and $B_{d-1}$, respectively. Without loss of generality we assume that $V_{B_{1}}=\left\{u_{1}, u_{2}\right\} \cup\left\{w_{1}, \ldots, w_{l}\right\}$. (We remark that possibly $V_{B_{1}}=\left\{u_{1}, u_{2}\right\}$.) Since $B_{0}$ is a simplex and the blocks $B_{0}$ and $B_{1}$ share a vertex, $B_{1}$ is not a simplex (by Claim 1), and therefore each of the vertices $w_{1}, \ldots, w_{l}$ is a cut vertex. Let $B_{1}^{\prime}, \ldots, B_{l}^{\prime}$ be blocks distinct from $B_{1}$ containing the vertices $w_{1}, \ldots, w_{l}$, respectively. The choice of $P$ implies that $B_{1}^{\prime}, \ldots, B_{l}^{\prime}$ are end blocks in $G$ and they are unique (by Claim 1). Let $H$ denote the subgraph of $G$ induced by the vertices belonging to the blocks $B_{0}, B_{1}^{\prime}, \ldots, B_{l}^{\prime}$. It is obvious that $H$ is a general corona $K_{m}^{k_{1}, \ldots, k_{m}}$, where $m=l+1, k_{1}=\left|V_{B_{0}}\right|-1, k_{2}=\left|V_{B_{1}^{\prime}}\right|-1, \ldots, k_{m}=\left|V_{B_{l}^{\prime}}\right|-1$. Let $G^{\prime}$ denote the subgraph $G-V_{H}$ of $G$. Since $G$ can be obtained from $G^{\prime}$ by (re)attaching the general corona $H$ to the vertex $u_{2}$ in $G^{\prime}$, it follows from the second part of Proposition 5 that $\alpha_{c}\left(G^{\prime}\right)=\alpha\left(G^{\prime}\right)$. Thus, since $G^{\prime}$ has $n^{\prime}$ blocks, where $1 \leq n^{\prime}<n, G^{\prime}$ belongs to $\mathcal{F}$ by the inductive hypothesis. Consequently, $G$ belongs to $\mathcal{F}$ (since $G$ can be obtained from $G^{\prime}$ by attaching a general corona to a vertex in $G^{\prime}$ ).

In the following theorem (which partially follows from Theorem 2) we prove the equivalent properties that characterize the trees $T$ with $\alpha(T)=\alpha_{\mathcal{c}}(T)$, that is, the $\alpha$ excellent trees.

Theorem 3. Let $T$ be a tree of order $n \geq 2$. Then the following statements are equivalent:

(a) $\alpha(T)=n / 2$.

(b) $T \in \mathcal{E}$.

(c) Thas a perfect matching.

(d) Thas a spanning forest in which every component is the corona of a tree.

(e) $T$ is an $\alpha$-excellent tree.

(f) $\quad \alpha(T)=\alpha_{c}(T)$.

Proof. The equivalence of (a) and (b) was proved in [9]. The statements (b), (e), and (f) are equivalent by Theorem 2. In [10] it was proved that (c) and (e) are equivalent. The statements (c) and (d) are equivalent: If $M$ is a perfect matching in $T$, then the subgraphs of $T$ generated by single edges belonging to $M$ form the desired forest (with the smallest number of edges). On the other hand if $T$ has a spanning forest $F$ in which every component is the corona of a tree, then the set of all pendant edges of $F$ forms a perfect matching of $T$. This completes the proof.

\section{Graphs $G$ with $i(G)=\alpha_{c}(G)$}

In this section we are interested in recognizing the structure of trees in which the independence number $i$ and the common independence number $\alpha_{c}$ are equal. In order to do this, we recall that a graph $G$ is a well-covered graph if every maximal independent set of vertices of $G$ is a largest independent set of $G$. Equivalently, $G$ is well-covered if and only if $i(G)=\alpha(G)$. We begin with some basic observations on well-covered graphs. The first one follows directly from the definition of a well-covered graph. The second one-the characterization of well-covered trees-was proved by Ravindra [11].

Remark 1. A graph $G$ is well-covered if and only if every set of independent vertices of $G$ is a subset of a largest independent set of $G$. 
Lemma 1 ([11]). A tree $T$ is well-covered if and only if it is $K_{1}$ or it is a corona of a tree.

In the next lemma we present a general property of the graphs $G$ for which $i(G)=\alpha_{c}(G)$.

Lemma 2. If $G$ is a graph in which $i(G)=\alpha_{c}(G)$, then either $i(G)=\alpha_{c}(G)=1$ or there is a vertex $z$ in $G$ such that $G-N_{G}[z]$ is a well-covered graph and $i\left(G-N_{G}[z]\right)=i(G)-1=$ $\alpha_{c}(G)-1=\alpha\left(G-N_{G}[z]\right)$.

Proof. Assume that $i(G)=\alpha_{c}(G) \geq 2$ and suppose that $G-N_{G}[x]$ is a non-well-covered graph for every $x \in V_{G}$. Then $i\left(G-N_{G}[x]\right)<\alpha\left(G-N_{G}[x]\right)$ for every $x \in V_{G}$, and consequently by Proposition 3 we have $i(G)=\min \left\{i\left(G-N_{G}[x]\right): x \in V_{G}\right\}+1<$ $\min \left\{\alpha\left(G-N_{G}[x]\right): x \in V_{G}\right\}+1=\alpha_{c}(G)$, a contradiction. From this contradiction and from the fact that $i\left(G-N_{G}[x]\right) \leq \alpha\left(G-N_{G}[x]\right)$ for every $x \in V_{G}$ it follows that $i\left(G-N_{G}[v]\right)=\alpha\left(G-N_{G}[v]\right)$ for some vertex $v$ of $G$, which in turn implies that $G-N_{G}[v]$ is a well-covered graph. We now claim that there is a vertex $z$ in $G$ such that $i(G)-1=$ $i\left(G-N_{G}[z]\right)=\alpha\left(G-N_{G}[z]\right)=\alpha_{c}(G)-1$. To observe this, let $z$ be a vertex such that $\alpha\left(G-N_{G}[z]\right)=\alpha_{\mathcal{C}}(G)-1$. Then, since $\alpha_{\mathcal{c}}(G)-1=i(G)-1 \leq i\left(G-N_{G}[z]\right) \leq$ $\alpha\left(G-N_{G}[z]\right)$, we also have $i(G)-1=i\left(G-N_{G}[z]\right)$ and this implies our claim.

Remark 2. Graphs in Figure 3 illustrate that if $G$ is a graph in which $i(G)=\alpha_{c}(G)$, then it follows from Lemma 2 that $G$ has at least one vertex $z$ (the white and solid black vertices) such that $G-N_{G}[z]$ is a well-covered graph, but only for some of them (the solid black vertices) the equalities $i\left(G-N_{G}[z]\right)=i(G)-1=\alpha_{c}(G)-1=\alpha\left(G-N_{G}[z]\right)$ hold.

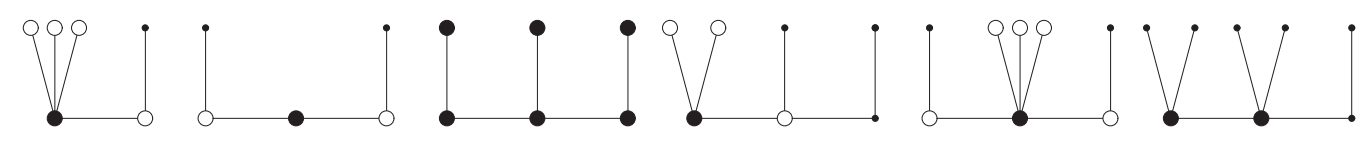

Figure 3. Graphs illustrating Remark 2.

We are now in position to present our characterization of trees for which the independence number $i$ and the common independence number $\alpha_{c}$ are equal.

Theorem 4. If $T$ is a tree, then $i(T)=\alpha_{\mathcal{c}}(T)$ if and only if at least one of the following conditions is fulfilled:

(1) $T$ is a star;

(2) $T$ is the corona of a tree;

(3) Thas a vertex $z$ such that

(a) $T-N_{T}[z]$ is a well-covered forest, and

(b) $\left|N_{T}(z) \cap L_{T}\right|>\sum_{v \in N_{T}(z)-L_{T}} \max \left\{\left|N_{T}(v) \cap\left(L_{T} \cup L_{T-N_{T}[z]}^{4}\right)\right|-1,0\right\}$ if $E_{T}\left(N_{T}(z)\right.$, $\left.L_{T} \cup L_{T-N_{T}[z]}^{4}\right) \neq \varnothing$, where $L_{T-N_{T}[z]}^{4}$ is the set of leaves of trees of order at least $4 \mathrm{in}$ $T-N_{T}[z]$.

Proof. Assume that $T$ is a tree and $i(T)=\alpha_{c}(T)$. It is obvious that if $i(T)=\alpha_{\mathcal{c}}(T)=1$, then $T$ is a star, $T=K_{1, n}$, where $n$ is a non-negative integer. Thus assume that $i(T)=\alpha_{c}(T) \geq 2$ and $T$ is not a corona graph. Then it follows from Lemma 2 that $T$ has a vertex $z$ such that $T-N_{T}[z]$ is a well-covered graph and $i\left(T-N_{T}[z]\right)=i(T)-1=\alpha_{c}(T)-1=\alpha(T-$ $\left.N_{T}[z]\right)$. Certainly, $T-N_{T}[z]$ is a well-covered forest, and, consequently, each component of $T-N_{T}[z]$ is the corona of a tree (that is, its pendant edges form a perfect matching, see $[7,11])$ or an isolated vertex.

Let $U$ and $V$ denote the set $N_{T}(z) \cap L_{T}$ and $N_{T}(z)-U$, respectively. We divide the set $V$ into two sets $V_{1}$ and $V_{2}$, where $V_{1}=\left\{v \in V: N_{T}(v) \cap\left(L_{T} \cup L_{T-N_{T}[z]}^{4}\right) \neq\right.$ $\varnothing\}$ and $V_{2}=V-V_{1}$. Let $F$ denote the graph $T-N_{T}[z]$, and let $c(F)$ be the set of all components of $F$. Let $F_{1}, F_{2}$, and $F_{12}$ be the subgraphs of $F$, where $F_{1}=\left\{H \in c(F):\left|V_{H}\right|=\right.$ 
1 or $\left(\left|V_{H}\right| \geq 4\right.$ and $\left.\left.N_{T}\left(V_{1}\right) \cap L_{H} \neq \varnothing\right)\right\}, F_{2}=\left\{H \in c(F):\left(\left|V_{H}\right|=2\right.\right.$ and $N_{T}\left(V_{2}\right) \cap$ $\left.V_{H} \neq \varnothing\right)$ or $\left(\left|V_{H}\right| \geq 4\right.$ and $\left.\left.N_{T}\left(V_{2}\right) \cap\left(V_{H}-L_{H}\right) \neq \varnothing\right)\right\}, F_{12}=\left\{H \in c(F):\left(\left|V_{H}\right|=\right.\right.$ 2 and $\left.N_{T}\left(V_{1}\right) \cap V_{H} \neq \varnothing\right)$ or $\left(\left|V_{H}\right| \geq 4\right.$ and $\left.\left.N_{T}\left(V_{1}\right) \cap\left(V_{H}-L_{H}\right) \neq \varnothing\right)\right\}$. By $Z_{1}, Z_{2}$, and $Z_{12}$ we denote the sets $N_{T}\left(V_{1}\right) \cap V_{F_{1}}, N_{T}\left(V_{2}\right) \cap V_{F_{2}}$, and $N_{T}\left(V_{1}\right) \cap V_{F_{12}}$, respectively. Figure 4 shows a tree $T$, the subsets $U, V_{1}, V_{2}, Z_{1}, Z_{2}, Z_{12}$, and the subgraphs $F_{1}, F_{2}$, and $F_{12}$ of the well-covered forest $F=T-N_{T}[z]$.

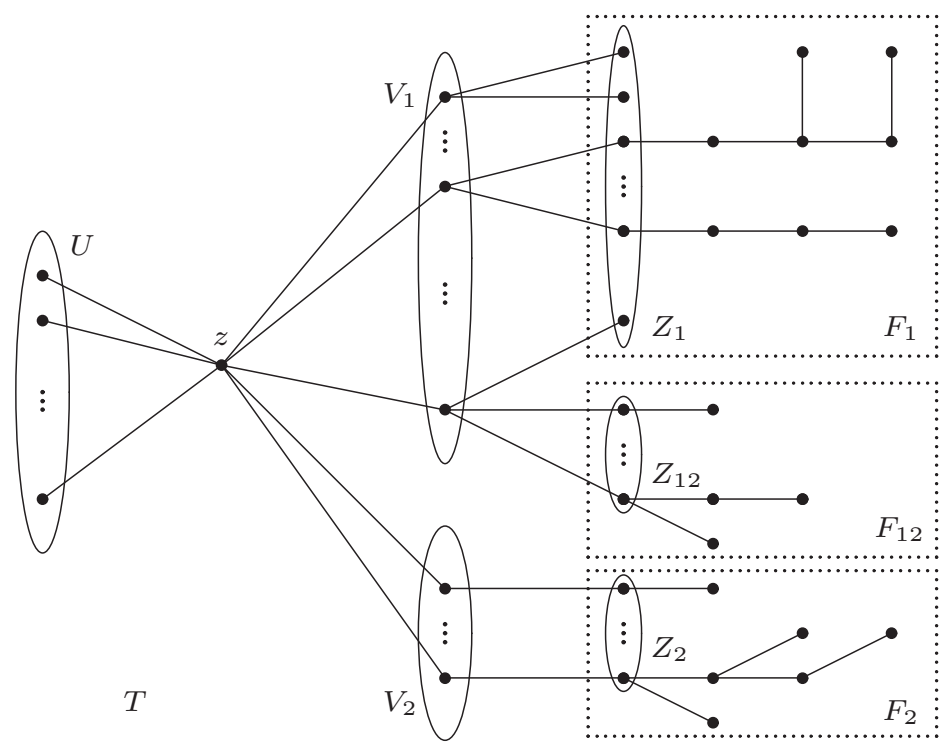

Figure 4. A tree $T$ for the proof of Theorem 4.

Assume that $E_{T}\left(N_{T}(z), L_{T} \cup L_{F}^{4}\right) \neq \varnothing$. Then the set $V_{1}$ is non-empty. Now, since $\max \left\{\left|N_{T}(v) \cap\left(L_{T} \cup L_{F}^{4}\right)\right|-1,0\right\}=\left|N_{T}(v) \cap\left(L_{T} \cup L_{F}^{4}\right)\right|-1$ if $v \in V_{1}$ and $\max \left\{\mid N_{T}(v) \cap\right.$ $\left.\left(L_{T} \cup L_{F}^{4}\right) \mid-1,0\right\}=0$ if $v \in V_{2}$, we have $\sum_{v \in N_{T}(z)-L_{T}} \max \left\{\left|N_{T}(v) \cap\left(L_{T} \cup L_{F}^{4}\right)\right|-1,0\right\}=$ $\sum_{v \in V_{1}}\left(\left|N_{T}(v) \cap\left(L_{T} \cup L_{F}^{4}\right)\right|-1\right)=\left|Z_{1}\right|-\left|V_{1}\right|$, and we shall prove that $|U|>\left|Z_{1}\right|-\left|V_{1}\right|$. Suppose to the contrary that $|U| \leq\left|Z_{1}\right|-\left|V_{1}\right|$. Let $I$ be a maximal independent set of $F$. Then $I \cup\{z\}$ is a maximal independent set of $T$ and therefore we have $i(T) \leq|I \cup\{z\}|=$ $i(F)+1$.

Now let $I_{1}, I_{2}$, and $I_{12}$ be sets such that $I_{1}$ is a maximal independent set of $F_{1}$ that contains $Z_{1} \cup\left\{x \in V_{F_{1}}: d_{T}\left(x, Z_{1}\right)=2\right\}, I_{2}$ is a maximal independent set of $F_{2}$ that contains $Z_{2}$, and $I_{12}$ is a maximal independent set of $F_{12}$ that contains $N_{T}\left(Z_{12}\right) \cap V_{F_{12}}$, respectively. The existence of such sets $I_{1}, I_{2}$, and $I_{12}$ follows from Remark 1 . Certainly, the set $I_{1} \cup I_{2} \cup I_{12}$ is a largest independent set of $F$. From the choice of $I_{1}, I_{2}$, and $I_{12}$ it is easy to observe that the set $\left(I_{1}-Z_{1}\right) \cup V_{1} \cup I_{2} \cup I_{12} \cup U$ is a maximal independent set of $T$. Thus, since $\left|Z_{1}\right|-\left|V_{1}\right|-|U| \geq 0$, we have $i(T) \leq\left|\left(I_{1}-Z_{1}\right) \cup V_{1} \cup I_{2} \cup I_{12} \cup U\right|=\left(\left|I_{1}\right|+\left|I_{2}\right|+\left|I_{12}\right|\right)-$ $\left(\left|Z_{1}\right|-\left|V_{1}\right|-|U|\right)=i(F)-\left(\left|Z_{1}\right|-\left|V_{1}\right|-|U|\right) \leq i(F)<i(F)+1=i(T)$, a contradiction which proves the desired inequality $|U|>\left|Z_{1}\right|-\left|V_{1}\right|$.

Assume that $T$ is a tree which has one of the properties (1), (2), and (3). It is evident that if $T$ is a star or a corona graph, then $i(T)=\alpha_{c}(T)$. Thus assume that $T$ is neither a star nor a corona graph, and $T$ has a vertex $z$ such that the subgraph $F=T-N_{T}[z]$ is a well-covered forest. Assume that $i(F)=\alpha(F)=\alpha_{c}(F)=m$. We shall prove that $i(T)=\alpha_{c}(T)=m+1$.

It is obvious that if $I$ is a maximal independent set of $F$, then $I \cup\{z\}$ is a maximal independent set of $T$ and therefore $i(T) \leq|I \cup\{z\}|=m+1$. On the other hand, if we assume that every maximal independent set of $T$ has at least $m+1$ vertices, then $m+1 \leq i(T)$, and, consequently, $i(T)=m+1$. This implies that $i(T)=\alpha_{c}(T)=m+1$ as $m+1=i(T) \leq \alpha_{c}(T)=\min \left\{\alpha(F): x \in V_{T}\right\}+1 \leq \alpha(F)+1=m+1$. Hence, to prove that $i(T)=\alpha_{\mathcal{c}}(T)=m+1$, it suffices to show that every maximal independent set of $T$ has at least $m+1$ vertices. (In fact, it suffices to show that every smallest maximal independent 
set of $T$ has at least $m+1$ vertices.) For this, let $U, V, V_{1}, V_{2}, Z_{1}, Z_{2}, Z_{12}, F, F_{1}, F_{2}$, and $F_{12}$ be the sets and graphs defined in the previous part of our proof.

Therefore assume that $J$ is a maximal independent set of $T$. If $J \cap V=\varnothing$, then the maximality of $J$ implies that the sets $J \cap V_{F}$ and $J \cap(U \cup\{z\})$ are non-empty, each of them is independent, and $J=\left(J \cap V_{F}\right) \cup(J \cap(U \cup\{z\}))$. In addition, the maximality of $J$ in $T$ implies that $N_{T}[x] \cap J \neq \varnothing$ for every $x \in V_{T}$. We shall prove that $J \cap V_{F}$ is a maximal independent set in $F$, that is, we shall prove that $N_{F}[x] \cap\left(J \cap V_{F}\right) \neq \varnothing$ for every $x \in V_{F}$. If $x \in V_{F}-\left(Z_{1} \cup Z_{2} \cup Z_{12}\right)$, then $N_{F}[x]=N_{T}[x] \subset V_{F}$ and therefore $N_{F}[x] \cap V_{F}=N_{T}[x]$ and, consequently, $N_{F}[x] \cap\left(J \cap V_{F}\right)=\left(N_{F}[x] \cap V_{F}\right) \cap J=N_{T}[x] \cap J \neq$ $\varnothing$. If $x \in Z_{1} \cup Z_{2} \cup Z_{12}$, then $N_{F}[x]=N_{T}[x]-V \subseteq V_{F}$ and, thus, $N_{F}[x] \cap\left(J \cap V_{F}\right)=$ $N_{F}[x] \cap J=\left(N_{T}[x]-V\right) \cap J=\left(N_{T}[x] \cap J\right)-(V \cap J)=N_{T}[x] \cap J \neq \varnothing$. This proves that $J \cap V_{F}$ is a maximal independent set in the well-covered graph $F$. Thus $\left|J \cap V_{F}\right|=m$ and finally $|J|=\left|J \cap V_{F}\right|+|J \cap(U \cup\{z\})| \geq m+1$. It remains to prove that $|J| \geq m+1$ if $J \cap V \neq \varnothing$. Thus assume that $J$ is a maximal independent set of $T$, where $J \cap V \neq \varnothing$. In this case $z \notin J$ and $U \subset J$. We distinguish two cases: $E_{T}\left(N_{T}(z), L_{T} \cup L_{F}^{4}\right)=\varnothing$, $E_{T}\left(N_{T}(z), L_{T} \cup L_{F}^{4}\right) \neq \varnothing$.

Case 1. Assume first that $E_{T}\left(N_{T}(z), L_{T} \cup L_{F}^{4}\right)=\varnothing$. In this case the sets $V_{1}, Z_{1}$, and $Z_{12}$ are empty, while $V=V_{2}$ and $V_{F}=V_{F_{2}}$ are non-empty (as $T$ is not a star). From the maximality of $J$ it follows that $J=\left(J \cap V_{F_{2}}\right) \cup\left(J \cap V_{2}\right) \cup U$, where $J \cap V_{2} \neq \varnothing$ (by our assumption). Certainly, $J \cap V_{F_{2}}$ is an independent set in $F_{2}$. We shall prove that $J \cap V_{F_{2}}$ is a maximal independent set of $F_{2}$. The maximality of $J$ implies that $N_{T}[x] \cap J \neq \varnothing$ for every $x \in V_{T}$. It remains to prove that $N_{F_{2}}[x] \cap\left(J \cap V_{F_{2}}\right) \neq \varnothing$ for every $x \in V_{F_{2}}$. If $x \in V_{2}-Z_{2}$, then $N_{F_{2}}[x]=N_{T}[x] \subseteq V_{F_{2}}$ and therefore $N_{F_{2}}[x] \cap\left(J \cap V_{F_{2}}\right)=N_{T}[x] \cap J \neq \varnothing$. It remains to prove that $N_{F_{2}}[x] \cap\left(J \cap V_{F_{2}}\right) \neq \varnothing$ if $x \in Z_{2}$. Assume that $x \in Z_{2}$. Then either $x$ is a non-leaf in $F_{2}$ or $x$ belongs to a component of order 2 in $F_{2}$, and, since $F_{2}$ is a well-covered forest, every component of $F_{2}$ is the corona of a tree, $x$ is adjacent to exactly one leaf in $T$, say to $\bar{x}$. Now, the maximality of $J$ in $T$ implies that the closed neighborhood $N_{T}[\bar{x}]=\{x, \bar{x}\}$ contains a vertex belonging to $J$ in $T$. This immediately implies that the closed neighborhood $N_{F_{2}}[x]$ contains a vertex belonging to $J \cap V_{F_{2}}$ in $F_{2}$. This completes the verification of the maximality of $J \cap V_{F_{2}}$ in $F_{2}$. Consequently, $\left|J \cap V_{F_{2}}\right|=m$ and $|J|=\left|\left(J \cap V_{F_{2}}\right) \cup\left(J \cap V_{2}\right) \cup U\right| \geq\left|J \cap V_{F_{2}}\right|+\left|J \cap V_{2}\right| \geq m+1$.

Case 2. Now assume that $E_{T}\left(N_{T}(z), L_{T} \cup L_{F}^{4}\right) \neq \varnothing$ and $|U|>\left|Z_{1}\right|-\left|V_{1}\right|$. This time the sets $U, V_{1}$, and $Z_{1}$ are non-empty. Let $J$ be a smallest maximal independent set of $T$ such that $J \cap V \neq \varnothing$. Then $z \notin J$ and $U \subset J$. We shall prove that $|J| \geq m+1$. Let us first observe that $J \cap V_{2}=\varnothing$. Suppose that $J \cap V_{2} \neq \varnothing$. Similarly as in Case 1 we can observe that $J \cap V_{F_{2}}$ is a maximal independent set of the well-covered graph $F_{2}$. The well-coveredness of $F_{2}$ implies that $F_{2}$ has a maximal independent set containing $Z_{2}$, say $J_{2}$ is such a set. Certainly, $\left|J_{2}\right|=\left|J \cap V_{F_{2}}\right|$ and, since $V_{2} \subset N_{T}\left(Z_{2}\right) \subseteq N_{T}\left(J_{2}\right)$, the set $\left(J-\left(V_{2} \cup V_{F_{2}}\right)\right) \cup J_{2}$ is a maximal independent set of $T$ and $\left|\left(J-\left(V_{2} \cup V_{F_{2}}\right)\right) \cup J_{2}\right|=|J|-\left|J \cap V_{2}\right|<|J|$, a contradiction. Consequently, $J \cap V_{2}=\varnothing$, and, therefore, $J \cap V_{1} \neq \varnothing$. We may assume that $J$ contains as many vertices belonging to $V_{1}$ as possible. Then, in fact, we may assume that $V_{1}$ is a subset of $J$ (for otherwise if $J_{1}$ were a maximal independent set of $F_{1}$ that contains $Z_{1} \cup\left\{x \in V_{F_{1}}: d_{T}\left(x, Z_{1}\right)=2\right\}$, and if $J_{12}$ were a maximal independent set of $F_{12}$ chosen in such a way that $N_{T}\left(Z_{12}\right) \cap V_{F_{12}} \subseteq J_{12}$ (the existence of such sets follows from Remark 1), then the set $J^{\prime}=\left(J-\left(V_{F_{1}} \cup V_{F_{12}}\right)\right) \cup\left(J_{1}-Z_{1}\right) \cup V_{1} \cup J_{12}$, for which $V_{1} \subset J^{\prime}$ and $\left|J^{\prime}\right| \leq|J|$ (as $\left|V_{1}\right| \leq\left|Z_{1}\right|$ ), would be a desired maximal independent set of $T$ ). Consequently, since $m=i(F)=i\left(F_{1} \cup F_{2} \cup F_{12}\right)=\left|J_{1}\right|+\left|J_{2}\right|+\left|J_{12}\right|$ and $J=\left(V_{1} \cup\left(J_{1}-Z_{1}\right)\right) \cup J_{2} \cup J_{12} \cup U$, we finally have $|J|=\left|V_{1}\right|+\left|J_{1}\right|-\left|Z_{1}\right|+\left|J_{2}\right|+\left|J_{12}\right|+|U|=\left(\left|J_{1}\right|+\left|J_{2}\right|+\left|J_{12}\right|\right)+(|U|-$ $\left.\left|Z_{1}\right|+\left|V_{1}\right|\right)=m+\left(|U|-\left|Z_{1}\right|+\left|V_{1}\right|\right) \geq m+1$. This completes the proof.

\section{Open Problems}

We know that $i(G) \leq \alpha_{c}(G) \leq \alpha(G)$ for every graph $G$ and we have characterized the trees $T$ for which $i(T)=\alpha_{c}(T)$ and the block graphs $G$ for which $\alpha_{\mathcal{c}}(G)=\alpha(G)$. In the light of these we have unsolved problems: 
1. Characterize different classes of graphs $G$ for which $i(G)=\alpha_{c}(G)\left(\alpha_{c}(G)=\alpha(G)\right.$, respectively).

2. Find lower and/or upper bounds for $\alpha_{\mathcal{C}}(G)$ for various classes of graphs $G$.

Author Contributions: Writing-original draft: M.D., M.L. and J.T. All authors contributed equally to this work. All authors have read and agreed to the published version of the manuscript

Funding: This research received no external funding.

Institutional Review Board Statement: Not applicable.

Informed Consent Statement: Not applicable.

Data Availability Statement: Not applicable.

Conflicts of Interest: The authors declare no conflict of interest.

\section{References}

1. Haynes, T.W.; Hedetniemi, S.T.; Slater, P.J. Fundamentals of Domination in Graphs; Chapman and Hall/CRC Pure and Applied Mathematics Series; Marcel Dekker, Inc.: New York, NY, USA, 1998.

2. Berge, C. Theory of Graphs and Its Applications; Methuen: London, UK, 1962.

3. Berge, C. Graphs and Hypergraphs; North-Holland: Amsterdam, The Netherlands, 1973.

4. Ore, O. Theory of Graphs; American Mathematical Society Colloquium Publications: Providence, RI, USA, 1962 ; Volume 38.

5. Goddard, W.; Henning, M.A. Independent domination in graphs: A survey and recent results. Discret. Math. 2013, 313, 839-854. [CrossRef]

6. $\quad$ Plummer, M.D. Some covering concepts in graphs. J. Comb. Theory 1970, 8, 91-98. [CrossRef]

7. Plummer, M.D. Well-covered graphs: A survey. Quaest. Math. 1993, 16, 253-287. [CrossRef]

8. Majeed, A.; Rauf, I. Graph theory: A comprehensive survey about graph theory applications in computer science and social networks. Inventions 2020, 5, 10. [CrossRef]

9. Domke, G.S.; Hatting, J.H.; Markus, L.R. On weakly connected domination in graphs II. Discret. Math. 2005, 305, 112-122. [CrossRef]

10. Fricke, G.H.; Haynes, T.W.; Hedetniemi, S.M.; Hedetniemi, S.T.; Laskar, R.C. Excellent trees. Bull. Inst. Comb. Appl. 2002, 34, 27-38.

11. Ravindra, G. Well-covered graphs. J. Comb. Inf. Syst. Sci. 1977, 2, 20-21. 